\title{
Optimization and Evaluation of Spinal Exoskeleton Design Concepts using Optimal Control
}

\author{
Monika Harant ${ }^{1}$, Matthias B. Näf ${ }^{2}$, Katja Mombaur $^{3}$ \\ ${ }^{1}$ Mathematics for the Digital Factory \\ Fraunhofer Intitute for Industrial Mathematics \\ Fraunhofer-Platz 1, 67663 Kaiserslautern, Germany \\ monika.harant@itwm.fraunhofer.de \\ ${ }^{2}$ Department of Mechanical Engineering \\ Vrije Universiteit Brussel \\ Pleinlaan 2, 1050 Brussels, Belgium \\ matthias.basil.naf@vub.be \\ ${ }^{3}$ CERC Human-Centred Robotics and Machine Intelligence \\ University of Waterloo \\ 200 University Avenue West, N2L 3G1 Waterloo, Canada \\ katja.mombaur@uwaterloo.ca
}

\begin{abstract}
Exoskeletons for the lower back are promising tools to support workers during heavy lifting tasks. Their development process faces several challenges. It is still not known which criteria the support must meet to prevent low-back pain and how users of different body stature and execution of lifting movements influence it. Thus, studying these factors needs an extensive testing on the human body and every considered design concept needs already a sophisticated prototype that subjects can wear for several hours. To overcome this issue, we propose a method using multibody dynamics and optimal control to optimize the design of an existing prototype (PO) as well as evaluate a new concept (DC) that incorporates motors at the hip joint. A dynamic model of the prototype with matching torque generation was developed, which also takes an approximation of possible misalignment into account. The human-robot interaction is simulated in an all-at-once approach that allows to calculate the muscle activity of the user required in addition to the exoskeleton support to reproduce recorded lifting motions. By minimizing the users' muscle activity, parameters describing the characteristics of the passive elements and, in case of DC, motor torque profiles are optimized. Compared to the initial setup, a significant improvement in exoskeletal support was achieved across all subjects in both cases while contact forces remained within prescribed limits to ensure a comfortable usage of the device. DC provides less support than PO but better control of the human-robot interaction.
\end{abstract}

Keywords: Optimal Control, Exoskeletons, Parameter Optimization, Multibody Dynamics, Biomechanics.

\section{INTRODUCTION}

Exoskeletons are receiving increasing attention not only in the medical field as a rehabilitation tool [1][2][3], but also in industry to improve working conditions [4][5][6]. Spinal exoskeletons are seen as a promising tool to assist workers with lifting tasks and reduce their muscle activity [5] [7], and thus their risk of low back pain. However, the development of wearable robots is challenging because the analysis of their effect on the human body as well as the assertion of a comfortable usage need an extensive testing of the device. This may take a long time and need a high number 
of prototypes. Thus, it is desirable to use optimization techniques to accelerate and facilitate this process. In [8], a method is presented to optimize the support of a lower body exoskeleton while it is worn by subjects. This method is difficult to apply to spinal exoskeletons because the subjects would have to lift objects repeatedly over a long period of time while the robot applies high forces on them.

In this work, we illustrate an alternative approach by using multibody dynamics and optimal control to optimize the passive elements of an existing prototype [9] in simulation as well as evaluating a new design concept (DC) that incorporates motors at the hip joint. Both the human and the exoskeleton are simulated in an all-at-once approach that allows the calculation of forces applied by the exoskeleton and the muscle activity needed by the user to reproduce a recorded lifting motion. Previous work applied this method successfully to a simple generic exoskeleton [10][11]. In [12], preliminary results optimizing the passive elements of the same prototype are presented. In addition to the new design concept DC, which also includes motors, we extended the method of [12] by including an estimation of the exoskeleton's torque generation taking misalignment with respect to the user into account.

In Sec. 2, a brief overview of the experiments performed for the reference lifting motions and for the misalignment approximation of the state of the passive elements is given. Then the models of the user and the exoskeleton applied in the optimal control problem are described in Sec. 3. The optimal control problem itself is formulated in Sec. 4. The results of the optimization and a discussion in Sec. 5 and 6 conclude this paper.

\section{EXPERIMENTS}

Data of two different biomechanical experiments were used in this work. In the following, a short summary of both is presented.

\subsection{Experiments for the Reference Lifting Motions}

Lifting motions performed by 5 male subjects (age $21-36$ years, weight $60-82 \mathrm{~kg}$, height 1.70 $-1.82 \mathrm{~m}$ ) were recorded. For the optimization, we use stoop-lifts of a $10 \mathrm{~kg}$ box with handles that was picked up from a $0.3 \mathrm{~m}$ high pedestal placed directly in front of the subjects. Subjects were instructed on the general form of a stoop-lift but were advised to perform the motion in a way that is comfortable for them, i.e. bending the knees was allowed.

The kinematics (marker positions) were measured at $44 \mathrm{~Hz}$ by an active motion capture system with two cameras (Certus Northern Digital Inc., Canada). The position of the box was also recorded using two marker clusters. Ground reaction forces of the subject and of the box were recorded at $1000 \mathrm{~Hz}$ by two force plates (Kistler Instrumente $\mathrm{GmbH}$, Suisse) and the forces between hands and box handles using uni-directional force sensors. During all trials, the muscle activity of the subjects was recorded using 12 EMG sensors.

For adjusting the models of the human actuation described in Sec. 3.1.3, it is useful to have an estimate of the maximum muscle activation observed during the recorded lifting motions. For this purpose, maximum voluntary contraction trials (MVC) were performed during the experiments with the aim to measure the EMG signal of the muscles when fully contracted. Two different MVC trials were used to measure the strength of the lumbar extensors and flexors as well as the hip extensors:

1. The subject stands on a force plate and performs a maximum effort deadlift against a fixed barbell.

2. The subject is restrained against an instrumented back rest using heavy Velcro straps and then having them extend, then flex their torso against the device.

For more information on this experiment, please refer to [11]. 


\subsection{Experiments for the Alignment between User and Exoskeleton}

Three young, healthy subjects (avg. age: 28 years, avg. height: $177 \mathrm{~cm}$, avg. weight: $71.3 \mathrm{~kg}$ ) performed a variety of tasks while wearing the prototype. We only consider the lifting tasks consisting of free lift, stoop lift, and squat lift. The tasks were performed with locked and unlocked misalignment mechanisms (three-revolute-joint-segment, and sliders, please refer to [9]). For our regression analysis described in Sec. 3.5, we only considered the recordings with unlocked configuration as this represents the standard behavior of the exoskeleton. Active markers were placed on the subject as well as the exoskeleton. Their positions were recorded using an active motion capture system with three cameras (Certus Optotrak, Northern Digital, Canada) at a frequency of $50 \mathrm{~Hz}$. The markers for the exoskeleton were attached to the slider connected to the beams, the part of the pelvis module where the beams are connected to, the passive element at the hip joint and to the slider of the thigh interface. The state of the exoskeleton was additionally recorded using its on-board sensors at a frequency of $100 \mathrm{~Hz}$. For further information, please refer to [13].

\section{APPLIED MODELS}

The recorded lifting motions used in the optimization are fairly symmetrical. This allows us to reduce the complexity of the system by modeling the human, exoskeleton, and box as symmetric rigid multibody systems in the sagittal plane (Fig. 1). First, a brief overview of the human model with muscle torque generators as joint actuators is given. Then the model of the exoskeleton prototype with its passive elements and the additional actuators for DC as well as the misalignment approximation is described.

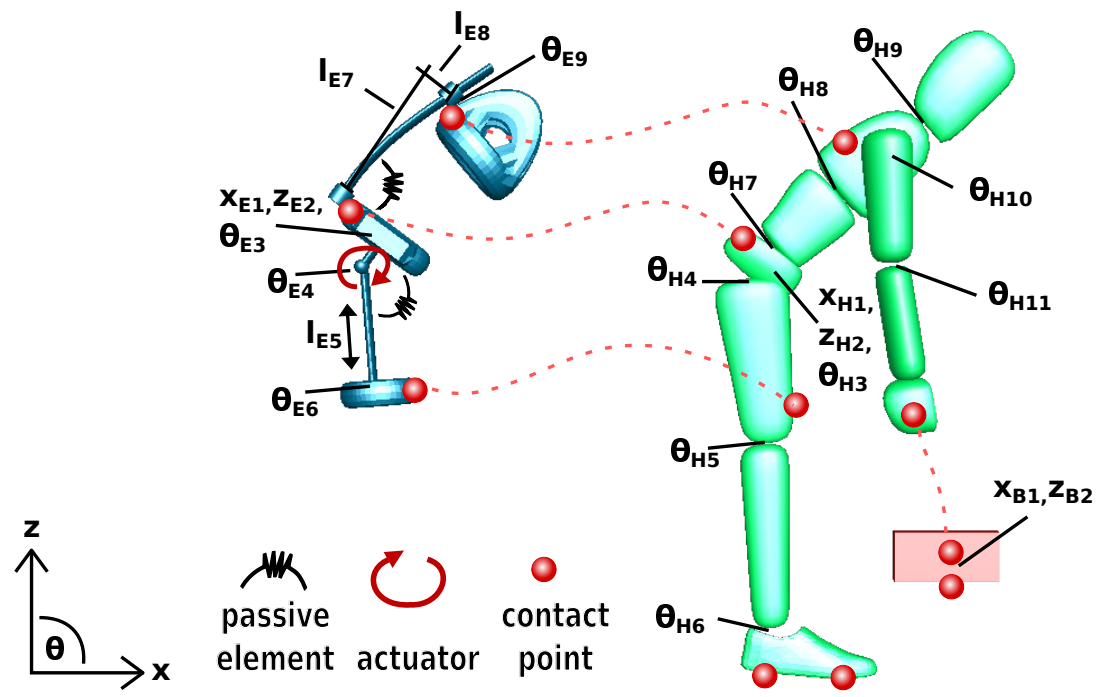

Figure 1. Modeled human, exoskeleton, and box with their respective degrees of freedom. The red dots show the locations where the box is connected to the ground and the human to the ground, the exoskeleton, and the box using contacts and loop closure constraints.

\subsection{Modeling the Human with Box}

The human model consists of 11 degrees of freedom (DoF) (Fig. 1). Both arms and legs are lumped together and the trunk is divided into three segments. Every human model was adjusted to represent the properties of each subject of the used motion capture recordings. The dynamic properties (segment mass, center of mass and inertia tensors) are estimated via regression equations proposed by de Leva [14] which are scaled by the height and weight of the person. In addition, the segment lengths of each subject were determined and the dynamic properties were linearly scaled accordingly. 


\subsubsection{Kinematic Fitting}

For the optimization, the recorded motions have to be transferred onto the models to obtain the respective joint positions. Each model segment was equipped with multiple virtual markers, which were positioned with the aid of a static trial to match the real markers on the subjects. Because of the marker placement as clusters, the joint positions $q \in \mathbb{R}^{11}$ are obtained by minimizing a combination of the distance between certain virtual and motion capture markers and the difference in the orientation of the marker clusters and model segments for each frame separately:

$$
\min _{q} \sum_{i \in N_{p}} a_{i}\left\|m_{i}^{V}(q)-m_{i}^{C}\right\|_{2}^{2}+\sum_{i \in N_{r}} b_{i}\left\|f_{\text {angle_axis }}\left(\left(R_{i}^{V}(q)\right)^{T} R_{i}^{C}\right)\right\|_{2}^{2}
$$

with $m_{i}^{V}(q)$ and $m_{i}^{C} \in \mathbb{R}^{3}$ the position of the virtual and motion capture markers. The matrices $R_{i}^{V}$ and $R_{i}^{C} \in \mathbb{R}^{3 \times 3}$ describe the orientation of the body segment and of the corresponding marker cluster in the global frame, respectively. $N_{p}$ and $N_{r}$ specify the set of marker indices whose position and whose orientation should be matched. The function $f_{\text {angle_axis }}(R)$ calculates the angle-axis representation of rotation matrix $R$. Each term is weighted by the factors $a_{i}$ and $b_{i} \in \mathbb{R}$. In Tab. 1, the accuracy of the kinematic fitting in terms of position and angle error is given.

Table 1. Average angle and position error and standard deviation with respect to the sagittal plane of the recorded and fitted model clusters of the five subjects.

\begin{tabular}{c||cc|cc} 
Subject & avg. angle error & {$[\mathrm{std}]$} & avg. position error & {$[\mathrm{std}]$} \\
\hline S1 & $2.20^{\circ}$ & {$[0.92]$} & $0.62 \mathrm{~cm}$ & {$[0.40]$} \\
S2 & $1.89^{\circ}$ & {$[0.89]$} & $0.28 \mathrm{~cm}$ & {$[0.22]$} \\
S3 & $2.82^{\circ}$ & {$[1.31]$} & $0.74 \mathrm{~cm}$ & {$[0.35]$} \\
S4 & $2.73^{\circ}$ & {$[1.50]$} & $1.00 \mathrm{~cm}$ & {$[0.82]$} \\
S5 & $2.67^{\circ}$ & {$[1.01]$} & $0.66 \mathrm{~cm}$ & {$[0.41]$}
\end{tabular}

\subsubsection{Muscle Torque Generators}

The human model is actuated by pairs of agonist and antagonist muscle torque generators (MTG) [15] with additional joint damping. One MTG summarizes the muscular properties for a joint in one direction (here: flexion and extension). Instead of having several line type muscles for bending a segment, only one MTG is needed, which greatly reduces the complexity of the problem without loosing much accuracy. The torque generated at the human joint $i$ is calculated by:

$$
\tau_{i}^{H U M A N}=\tau_{i}^{F L}+\tau_{i}^{E X}+\beta_{i} \dot{q}_{i} \quad \text { with } \beta_{i}=\eta_{i} \frac{{ }^{i} \tau_{0}^{F L}+{ }^{i} \tau_{0}^{E X}}{{ }_{i} \omega_{\max }^{F L}+{ }^{i} \omega_{\max }^{E X}} .
$$

The torque generated by the MTG for flexion and extension is denoted by $\tau^{F L}$ and $\tau^{E X}$, respectively. The joint velocities is given by $\dot{q}$. For each MTG, $\tau_{0}$ is the maximum isometric torque and $\omega_{\max }$ the maximum angular velocity. The damping coefficient $\beta$ is scaled by factor $\eta$.

The amount of torque a muscle can produce depends on the level of activation, the joint position and the joint velocity. This dependency is modeled in the MTG by three curves: the active $\left(f^{A}\right)$ and passive $\left(f^{P}\right)$ torque - angle curve representing the active and passive forces generated by the muscles and the curve $f^{V}$ describing their torque - velocity relationship:

$$
\tau^{M T G}=\tau_{0}^{M T G}\left[\alpha f^{A}(\theta) f^{V}(\omega)+s^{P} f^{P}\left(\theta-\theta_{0}^{P}\right)\left(1-\beta^{P} \frac{\omega}{\omega_{\max }^{M T G}}\right)\right]
$$

with muscle activation $\alpha \in[0,1]$ and nonlinear normalized damping term $\beta^{P}$. The parameters $s^{P}$ and $\theta_{0}^{P}$ scale and shift the passive torque-angle-curve. The MTG-specific angle $\theta$ and velocity $\omega$ are derived from the joint angle $q$ and velocity $\dot{q}$, respectively. It should be noted that only uniarticular muscles are currently supported by the MTG. Thus, the effect of biarticular muscles in the movement performance is neglected during simulation. Further information regarding the MTG can be found in [10] [15]. 


\subsubsection{Muscle Torque Generator Fitting}

The possible strength of a MTG at a given position and velocity can be adjusted by a set of parameters. For the optimization, it is vital that the muscle models can generate the torques necessary to perform the motion. This is not guaranteed when using the default properties derived from experimental data of the literature as every person differs in strength and flexibility.

A muscle-fitting-routine [15] was applied to adjust the MTG so that they can reproduce the motion with muscle activation not exceeding a given range $\left[0, \alpha_{\max }\right]$. As the subject likely would not need their full strength to lift a $10 \mathrm{~kg}$ heavy box, an educated guess for the maximum activation level during the lifting motion would be helpful to get a more accurate representation of the muscle group properties for each joint. For this purpose, the available EMG data of the recorded lifting motions were normalized and its peak value was taken as activation limit $\left(\alpha_{\max }\right)$ of the MTG responsible for hip and lumbar extension during the muscle fitting process:

$$
E M G_{\text {trial }}^{\text {norm }}(t)=\frac{E M G_{\text {trial }}(t)-\overline{E M G_{\text {quiet }}}}{E M G_{M V C}^{M A X}-\overline{E M G_{\text {quiet }}}}
$$

with $E M G_{\text {trial }}$ the EMG data of the lifting trial, $\overline{E M G_{\text {quiet }}}$ the mean of the EMG data during quiet standing phase, and $E M G_{M V C}^{M A X}$ the peak value observed during a set of MVC trials explained in Sec. 2. In case no EMG data was available, 0.7 was taken as $\alpha_{\max }$ which is a conservative guess for athletic subjects lifting a $10 \mathrm{~kg}$ heavy box.

\subsection{Modeling the Exoskeleton}

The exoskeleton model is reduced to the sagittal plane like the human model. It has 9 DoF (6 internal) and consists of several modules: pelvis module, thigh bar, thigh interface, torso bar (set of beams) and torso interface, which are illustrated in Fig. 1. Between pelvis module and torso interface and between thigh bar and thigh interface, there are prismatic joints representing the deflection of the beams and the sliders on the prototype, and there are rotational joints to align the exoskeleton with the human model at specific contact points. The dynamic parameters (mass, center of mass, and inertia) were derived from CAD models of the existing prototype. The total mass of the exoskeleton is $6.7 \mathrm{~kg}$. It generates torques at the lower back by 3 carbon fiber beams and at the hip joint by passive elements with a nonlinear torque-angle relationship [16]. Mathematical models replicating their behavior are included in the optimization problem and are described in the next chapters. For DC, a motor is attached to the hip joint to evaluate their effect on the support and contact forces between user and exoskeleton which affects their alignment. The weight of the pelvis module is increased by $3 \mathrm{~kg}$ to account for the additional actuators.

\subsection{Modeling the Passive Hip Element}

The passive hip element [16] consists of a linear spring where a cable is attached to. The cable is guided through a system of rollers and attached to a heart-shaped profile at the center of a revolute joint. When the joint rotates, the cable is bend over the profile which leads to a nonlinear torque-angle relationship. The linear spring itself can be pretensioned. The following 6 parameters specify the shape of the torque profile:

$(S)$ linear spring stiffness, $(P)$ linear spring pretension, $\left(P_{\max }\right)$ maximum pretension of the spring, $(B)$ length of the lever arm, $(C)$ distance between rollers and cable attachment point at the joint, $(R)$ radius of the heart - shaped profile. Except for $(S)$ and $\left(P_{\max }\right)$, all parameters are later optimized. The torque $\tau$ generated at deflection angle $\alpha$ is derived as follows:

$$
\tau=\operatorname{sign}(\alpha) S\left(-C+B+D+E+P P_{\max }\right) J,
$$

with

$$
\begin{aligned}
D & =R \lambda, & E & =\sqrt{A^{2}-R^{2}}, & J & =C \sin (\lambda-|\alpha|), \\
A & =\sqrt{I^{2}+H^{2}}, & I & =C \cos (\alpha)-B, & H & =R-C \sin (|\alpha|), \\
\gamma & =\arctan \left(\frac{H}{I}\right), & \theta & =\arctan \left(\frac{E}{R}\right), & \lambda & =\frac{\pi}{2}-\gamma-\theta,
\end{aligned}
$$


Because of geometrical reasons, following constraint must hold so that the profile is fabricable:

$$
E>0
$$

The torque profile is non smooth for $\alpha=0$, which is reflected in the absolute function. In order to obtain a smooth function, the absolute function was approximated with

$$
|\alpha|_{\text {approx }}:=2 \alpha\left(\frac{1}{1+e^{-100 \alpha}}\right)-\alpha
$$

\subsection{Modeling the Carbon Fiber Beam}

The beam is modeled as a long thin cantilever beam (Fig. 2) having a uniform circular cross section made of a linear elastic material which is homogeneous and isotropous. A force $F$ is applied at its free end in a direction specified by the angle $\alpha \in \mathbb{R}$. As the lifting movement is reduced to the sagittal plane, only sagittal deflections of the beam are considered as well.

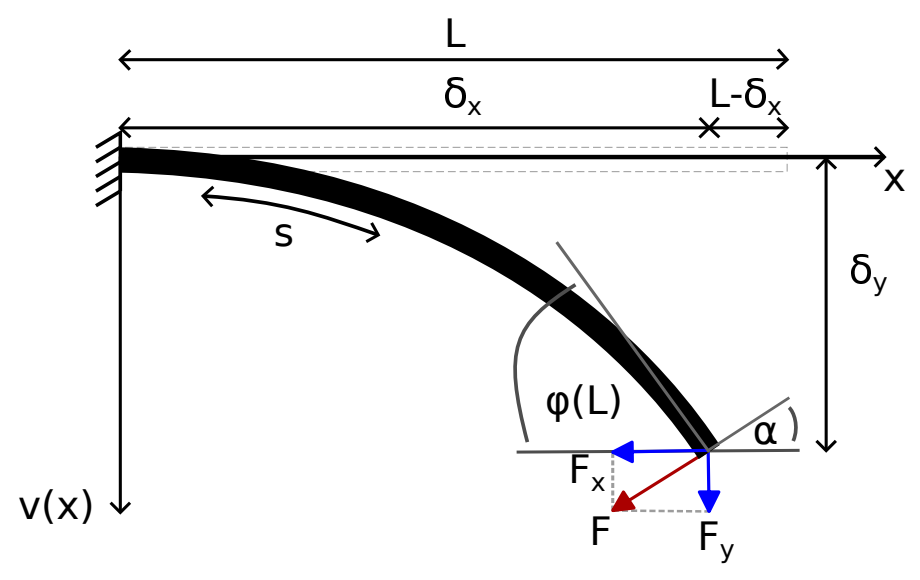

Figure 2. Cantilever beam

Assuming that the Bernoulli-Euler hypothesis is valid and applying the analysis proposed by [17] on the deflection of a cantilever beam, the Bernoulli-Euler bending moment-curvature relationship for this type of beam at a point $s$ along the beam with Cartesian coordinates $(x, y)$ (Fig. 2) can be formulated as follows:

$$
E I \frac{d \phi}{d s}=M(s), \quad \text { with } \quad M(s)=F\left[\sin (\alpha)\left(\delta_{x}-x\right)+\cos (\alpha)\left(\delta_{y}-v(x)\right)\right],
$$

where $M$ and $\kappa=\frac{d \phi}{d s}$ are the bending moment and curvature, respectively. The moment of inertia of a beam with circular cross section specified by radius $r$ is denoted by $I=\frac{\pi}{4} r^{4}$. The horizontal and vertical displacement at the end of the beam are given by $\delta_{x}$ and $\delta_{y}$ and $\alpha$ is the angle between the direction of the force and the neutral position of the beam when no forces are applied. Between the curvature $\frac{d \phi}{d s}$ of the deformed beam and the transverse displacement $v(x)$ following relationship can be established:

$$
\frac{d \phi}{d s}=\frac{\frac{d^{2} v}{d x^{2}}}{\left[1+\left(\frac{d v}{d x}\right)^{2}\right]^{\frac{3}{2}}} .
$$

By differentiating eq. 9 with respect to $\mathrm{s}$ and taking into account the relations $\frac{d x}{d s}=\cos (\phi)$ and $\frac{d y}{d s}=\sin (\phi)$, we obtain:

$$
E I \frac{d^{2} \phi}{d s^{2}}+F \sin (\alpha+\phi)=0 .
$$


This equation together with $\frac{d x}{d s}=\cos (\phi)$ and $\frac{d y}{d s}=\sin (\phi)$ can be integrated and solved for $F$ and $L$ when taking the following boundary conditions into account:

$$
v(0)=0, \quad v\left(\delta_{x}\right)=d_{y}, \quad \phi(0)=0, \quad \frac{d \phi}{d s}(L)=0,
$$

with $L$ the beam length. Note that in our case, the horizontal and vertical displacement of the beam is known whereas the generated force and the length of the beam are unknown as the connector of the torso interface can move freely on the slider along the beam.

\subsubsection{Polynomial Approximation of the Beam Deflection}

The above described method includes solving a boundary value problem which is too complex to be included in the optimal control setup described in Sec. 4. To avoid the integration of the system, we approximate the deflection of the beam by a polynomial $v(x) \approx P(x)=\sum_{i=0}^{N} a_{i} x^{i}$ of order $N \in \mathbb{N}$. The coefficient of the polynomial can be reduced by applying the boundary conditions $v(0)=0$ and $\frac{d v}{d x}(0)=0$ yielding $a_{0}=a_{1}=0$. The remaining coefficients and the force $F$ are computed by minimizing the distance to the deflection $\delta_{y}$ at the end and the deviation to the deflection-moment relationship (9) at equally spaced gridpoints $0=x_{0}<x_{1}<\cdots<x_{M}=\delta_{x}, M \in \mathbb{N}$. By setting $M=N$ the problem reduces to solving following set of equations:

$$
\begin{aligned}
P\left(\delta_{x}\right) & =\delta_{y} \\
P^{\prime \prime}\left(x_{k}\right) & =\left[1+P^{\prime}\left(x_{k}\right)^{2}\right]^{\frac{3}{2}} M\left(x_{k}\right)
\end{aligned}
$$

with $M\left(x_{k}\right)=\frac{F}{E I}\left(\sin (\alpha)\left(\delta_{x}-x_{k}\right)+\cos (\alpha)\left(\delta_{y}-P\left(x_{k}\right)\right), \quad x_{k}=\frac{k}{N} \delta_{x}, \quad k=0, \ldots, N-1\right.$

Based on the results using different order $N$ (Tab. 2), $N=7$ was used in the optimal control setup and the characteristics of the beam are optimized by adjusting the radius $r$ of the beam cross section.

Table 2. Deviation (mean [std]) of the force acting at the beam end calculated by the boundary value problem and the polynomial approximation of different order $N$ setting $\alpha=90^{\circ}, \delta_{x}=$ $0.4 \cos (x), \delta_{y}=0.4 \sin (x), x=0, \ldots, 50^{\circ}$

\begin{tabular}{c|c|c|c|c|c|c}
$N$ & 3 & 4 & 5 & 6 & 7 & 8 \\
\hline Deviation (N) & $2.9[4.1]$ & $1.1[2.6]$ & $0.3[0.7]]$ & $0.1[0.2]$ & $0.03[0.05]$ & $0.03[0.06]$
\end{tabular}

\subsection{Coupling of Human and Exoskeleton}

In the simulation, a rigid coupling between human and exoskeleton model is assumed, which is realized over loop closure constraints. This assumption does not fully reflect reality as there is noticeable movement between user and exoskeleton. However, to model a realistic behavior of the interaction, adequate experimental data from the prototype is needed which was not available. In the experiment presented in Sec 2.2, the movement of the arms and head and the position of the box were not recorded, making the data unsuitable for contact model identification using full-body simulations.

However, they provide an analysis on how the deflection of the human torso and thigh is different from the deflection of the exoskeleton's torso and thigh interface. This data was used to approximate the state of the passive elements with a linear regression model. Parameter values $p_{T}^{C}, p_{T}^{T}$, $p_{T}^{H}, p_{H}^{C}, p_{H}^{T}$ and $p_{H}^{H} \in \mathbb{R}$ were identified so that the values for the deflection of the torso module connector (or the beams) $\hat{\theta}_{T}^{E}\left(\equiv \operatorname{atan}\left(l_{E 8} / l_{E 7}\right)\right)$ and the exoskeleton hip joint angle $\hat{\theta}_{H}^{E}\left(\equiv \theta_{E 4}\right)$ obtained by

$$
\begin{aligned}
& \hat{\theta}_{T}^{E}=p_{T}^{C}+p_{T}^{T} \theta_{T}^{H}+p_{T}^{H} \theta_{H}^{H} \\
& \hat{\theta}_{H}^{E}=p_{H}^{C}+p_{H}^{T} \theta_{T}^{H}+p_{H}^{H} \theta_{H}^{H}
\end{aligned}
$$


match the experimental data best in the least-squares sense with $\theta_{T}^{H}\left(\equiv \theta_{H 7}+\theta_{H 8}\right)$ the human torso flexion angle and $\theta_{H}^{H}\left(\equiv \theta_{H 4}\right)$ the human hip flexion angle (see Fig. 1). In Tab. 3, the optimized linear regression parameters can be obtained and the results show that a significant linear regression relationship exists between the human and exoskeleton deflection angles.

Table 3. Linear regression parameters for given angles in radian with an error variance of $0.0044\left(R^{2}: 0.9616\right.$, p-value: 0.0$)$ for $\hat{\theta}_{T}^{E}$ and $0.0043\left(R^{2}: 0.9829, \mathrm{p}\right.$-value: 0.0$)$ for $\hat{\theta}_{H}^{E}$

\begin{tabular}{c|c|c||c|c|c}
$p_{T}^{C}$ & $p_{T}^{L}$ & $p_{T}^{H}$ & $p_{H}^{C}$ & $p_{H}^{T}$ & $p_{H}^{H}$ \\
\hline-0.0426 & 0.4082 & -0.3145 & -0.0017 & -0.2177 & 0.6912
\end{tabular}

Furthermore, several studies emphasized as an important requirement for exoskeletons to be comfortable to wear over hours even though they are tightly connected to the user with straps and plates and exert strong pushes and pullings [18] [4]. We address this issue by limiting the interaction forces between user and exoskeleton. These limits are based on [19] where several subjects were exposed to constant and repeating pressure and they graded in both cases at which level it was uncomfortable for them and at which level they felt pain. The average values for discomfort (Tab. 4) are used as limits in the optimization.

Table 4. Contact force limits based on the findings of [19] and a friction coefficient of 0.6

\begin{tabular}{l||c|c|c} 
Force Limit & Pelvis Contact & Thigh Contact & Torso Contact \\
\hline Normal Force & $162.40 \mathrm{~N}$ & $333.40 \mathrm{~N}$ & $230.30 \mathrm{~N}$ \\
Shear Force & $97.44 \mathrm{~N}$ & $200.04 \mathrm{~N}$ & $138.18 \mathrm{~N}$
\end{tabular}

\section{OPTIMAL CONTROL PROBLEM FORMULATION}

The lifting motion of the human model wearing the exoskeleton is set up as a 3-phase optimal control problem (OCP). In the first phase, the user stands in an upright position, bends down and makes contact with the box. In the second phase, the user generates enough force to lift the box. The last phase starts when the box leaves the ground and ends when the user holds the box in an upright position. The OCP is formulated as follows:

$$
\begin{array}{rlrl}
\min _{q, \dot{q}, z, \alpha, u, p} \Psi[q, \dot{q}, z, \alpha, u, p]:=\sum_{i=1}^{3}\left(\sum_{n=0}^{N_{i}}\left\|W_{q}\left(q\left(t_{i, n}\right)-q_{i, n}^{R E F}\right)\right\|^{2}+\int_{t_{i}}^{t_{i+1}} \phi(q, \dot{q}, z, \alpha, u, p) d t\right) & \\
\text { s.t. } \quad & & & \\
M(q) \ddot{q}+G_{i}(q)^{T} \lambda & =\tau(q, \dot{q}, z, \alpha, u, p)-C(q, \dot{q}) & & \\
\dot{\alpha} & =\left(\left(u_{m}-\alpha_{m}\right) / T_{m}\right)_{m=1, \ldots, N_{m}} & & \\
f(q, z, p) & =0 & & i=1, \ldots, 3
\end{array}
$$

with $q, \dot{q}$, and $\ddot{q}$ the joint positions, velocities, and accelerations, respectively. The number of shooting nodes of phase $i$ is denoted by $N_{i}$. The motion to be tracked is given for time $t_{i, n}$ by the joint positions $q_{i, n}^{R E F}$ and the fitting accuracy is specified by a weighting matrix $W_{q}$. The algebraic states $z$ and the system of equations (21) define the state of the beam. The parameters $p$ describe the design of the passive elements of the exoskeleton. The controls $u$ are the neural excitation of the MTG. In case of DC, the torque profile of the motors is a control as well. Eq.(20) are the MTG activation dynamics proposed by [20] with activation level $\alpha$ and (de-)activation time constant $T$. The number of MTG is given by $N_{m}$. The equation of motion of the constrained multibody system is given by (19) with mass matrix $M$, constraint jacobian $G_{i}$, and unknown force variables $\lambda$. The function $C$ contains the centrifugal, gravitational and Coriolis forces. The generalized forces are 
denoted by $\tau$ consisting of the joint torques and forces generated by the MTG and the exoskeleton. The Constraints (22) include, among others, position constraints, constraints on hand-to-box, boxto-floor, and foot-to-floor contact forces, human/exoskeleton alignment regulations and limits on parameters, states and controls. The objective function (18) consists of a least squares term for tracking the recorded motion and a Lagrange term enforcing the reduction of human joint moments and pelvis contact moment. The OCP is discretized using direct multiple shooting and the resulting NLP is solved with SQP and active-set method provided by the toolbox MUSCOD-II [21]. For the rigid multibody dynamics calculations, the open-source library RBDL [22] was used.

\section{RESULTS}

The support of the exoskeleton was optimized for several recorded lifting motions. For the prototype optimization (PO), parameters describing the the behavior of the beam (beam cross section radius) and the passive elements (see Sec. 3.3) were optimized. For the new design concept (DC), motor torque profiles were optimized as well. The results are compared to the original configuration $(\mathrm{O})$ of the prototype. The weighting of the cost function enforced a high fitting accuracy (avg. joint angle error across all subjects $0.17^{\circ}-0.62^{\circ}$ ) with the same values for all simulation setups $(\mathrm{O} / \mathrm{PO} / \mathrm{DC})$. This guarantees that the reduction in muscle activity comes solely from the support provided by the exoskeleton and not from an alteration of the motion.

In Tab. 5, the achieved support for each subject with respect to each setup is listed. Both optimized configurations, $\mathrm{PO}$ and $\mathrm{DC}$, provide higher support than $\mathrm{O}$ across all subjects. However, PO increases the support by additional $2.5-4.1 \%$ regarding the lumbar moment, $1.6-2.7 \%$ regarding the hip moment, and 1.9 - $3.7 \%$ regarding the peak lumbar moment compared to DC. The higher support of $\mathrm{PO}$ is based on torque profiles of the beams and $\mathrm{PH}$ with higher peaks than of DC during the motion (Fig. 3). Through the motors, a broader torque curve is achieved resulting in smaller loss of support at the hip joint than at the lumbar joint compared to PO. The fact that the limit on the normal contact force at the pelvis is reached for both PO and DC (Fig. 4) suggests that due to the higher weight of the pelvic module, a force/torque generation of the exoskeleton of DC at the same level as PO was not possible before the limit was reached.

The higher weight is also reflected in the shear forces acting at the pelvis contact point (Fig. 4), in particular at the beginning and end of the motion when the user is standing upright and the weight of the exoskeleton mainly contributes to them. The normal and shear forces of the thigh (max. 127 $\mathrm{N}$ and $7 \mathrm{~N}$ across subjects and PO/DC) and torso (max. $121 \mathrm{~N}$ and $16 \mathrm{~N}$ ) contact point stayed far below the set limits. As intended, adding the motor to the prototype resulted in a high reduction of the moment acting at the pelvis contact compared to PO (Fig. 4), staying within [-4.9 Nm, 3.6 Nm] in contrast to [-11.4 Nm, $5.4 \mathrm{Nm}]$ and yielding a very good alignment of exoskeleton and user. For comparison the contact moment of $\mathrm{O}$ lies in the range of $[-8.1 \mathrm{Nm}, 7.4 \mathrm{Nm}]$ for all subjects.

Table 5. Reduction of hip and lumbar moment when using the exoskeleton of DC I PO I O with respect to the corresponding human-only-simulation

\begin{tabular}{c|r|r|r|r}
\hline Subject & lumbar moment reduction* & \multicolumn{1}{|c|}{ hip moment reduction* } & peak lumbar moment reduc. \\
\hline S1 & $15.5 \%|18.7 \%| 14.1 \%$ & $12.1 \%|14.3 \%| 11.2 \%$ & $19.9 \%|23.5 \%| 17.5 \%$ \\
S2 & $13.5 \%|16.5 \%| 11.9 \%$ & $12.4 \%|14.1 \%| 10.1 \%$ & $13.4 \%|16.8 \%| 12.0 \%$ \\
S3 & $12.0 \%|15.4 \%| 10.7 \%$ & $7.7 \%|9.6 \%| 6.5 \%$ & $12.0 \%|15.5 \%| 10.6 \%$ \\
S4 & $14.3 \%|16.8 \%| 9.5 \%$ & $10.0 \%|11.6 \%|$ & $4.9 \%$ & $12.2 \%|14.1 \%| 7.6 \%$ \\
S5 & $18.6 \%|22.7 \%| 17.3 \%$ & $12.2 \%|14.9 \%| 11.3 \%$ & $16.3 \%|20.0 \%| 14.9 \%$ \\
\hline
\end{tabular}

* reduction in terms of the integrated area under the moment curve 

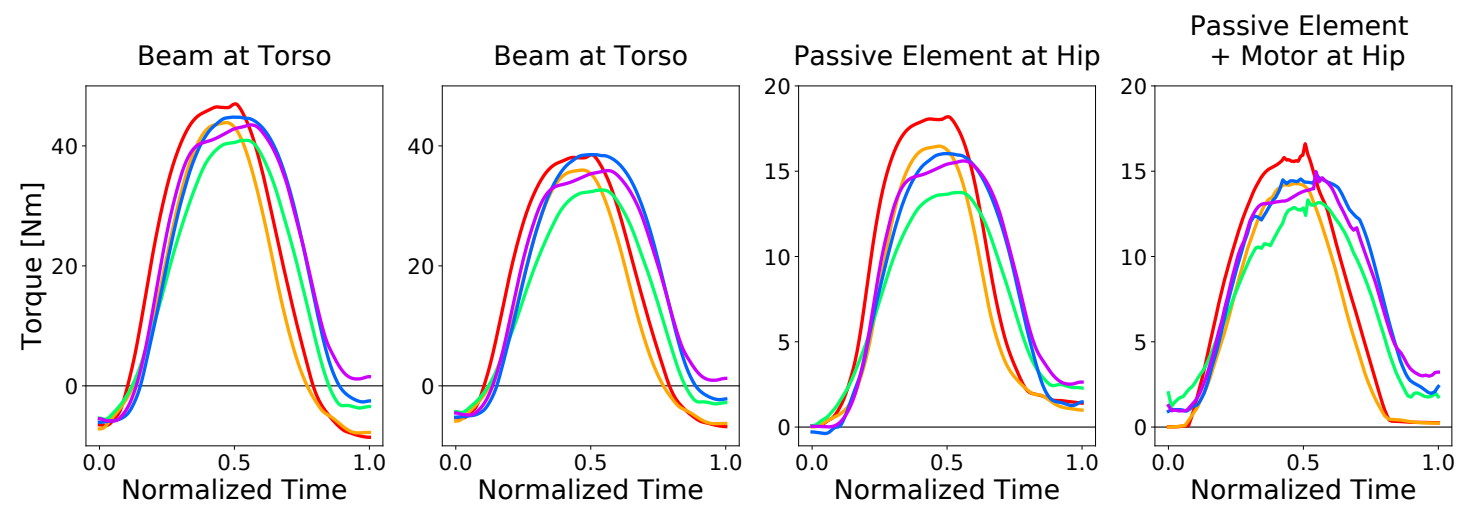

Figure 3. From left to right: The torques generated at the bottom of the beams (at lumbar joint level) during the motion of PO and of DC. The torques generated by PH during the motion using the exoskeleton configuration of PO and of DC. Please refer to Fig. 4 for the color legend regarding the subjects.

\section{DISCUSSION AND CONCLUSIONS}

In this work, we present a method to optimize and evaluate design concepts for exoskeletons in terms of contact forces between user and exoskeleton and load reduction using an optimal control formulation. It can be used to optimize the design of passive elements described by multiple parameters and motor torque profiles that serve as guidelines for the next prototype. The modular setup offers an easy switching between exoskeleton models. It can also be extended to perform optimization without reference data if the tracking term is left out, which was shown in [10]. Note that these calculations can also be done without having a real prototype and do not need biomechanical experiments involving long usages of the device as in [8].

It also provides an analysis of the actuation patterns of the user, which were not presented here but need to be considered during the optimization. Lifting motions involve going to the limits of the range of motion when also passive muscle forces are present. Thus, it is important to have an estimation of the distribution of active and passive muscle forces during simulation as the exoskeleton should only take over the active muscle part. Otherwise, the user may have to work against the exoskeleton while performing the same motion.

We analyzed the effect of including additional motors at the hip joint of an existing prototype having only passive elements and showed that this would lead to a decrease in the possible support while the contact moment acting at the pelvis can be much better controlled. One limitation is the assumption of rigid coupling between the user and the exoskeleton. In reality, movements occur with respect to each other. In context of the prototype evaluated, a movement or rotation of the exoskeleton segments with respect to the user may lead to a higher deflection of the beam resulting in a different, possible higher, support. We included an estimate of the effect the movement of the exoskeleton has on the passive elements using linear regression equations obtained by experimental data. A comparison to preliminary results presented in [12], showed that the simulated support in this work agrees better with the values observed in [5]. However, the regression equations only depend on the user's position and not on the generated torque distribution of the exoskeleton. In order to accurately model the human-robot interaction, additional experimental data is needed, which was not available for these calculations and is part of future work.

\section{ACKNOWLEDGMENTS}

This work was funded by the European Commissions within the H2020 project SPEXOR (GA 687662). 

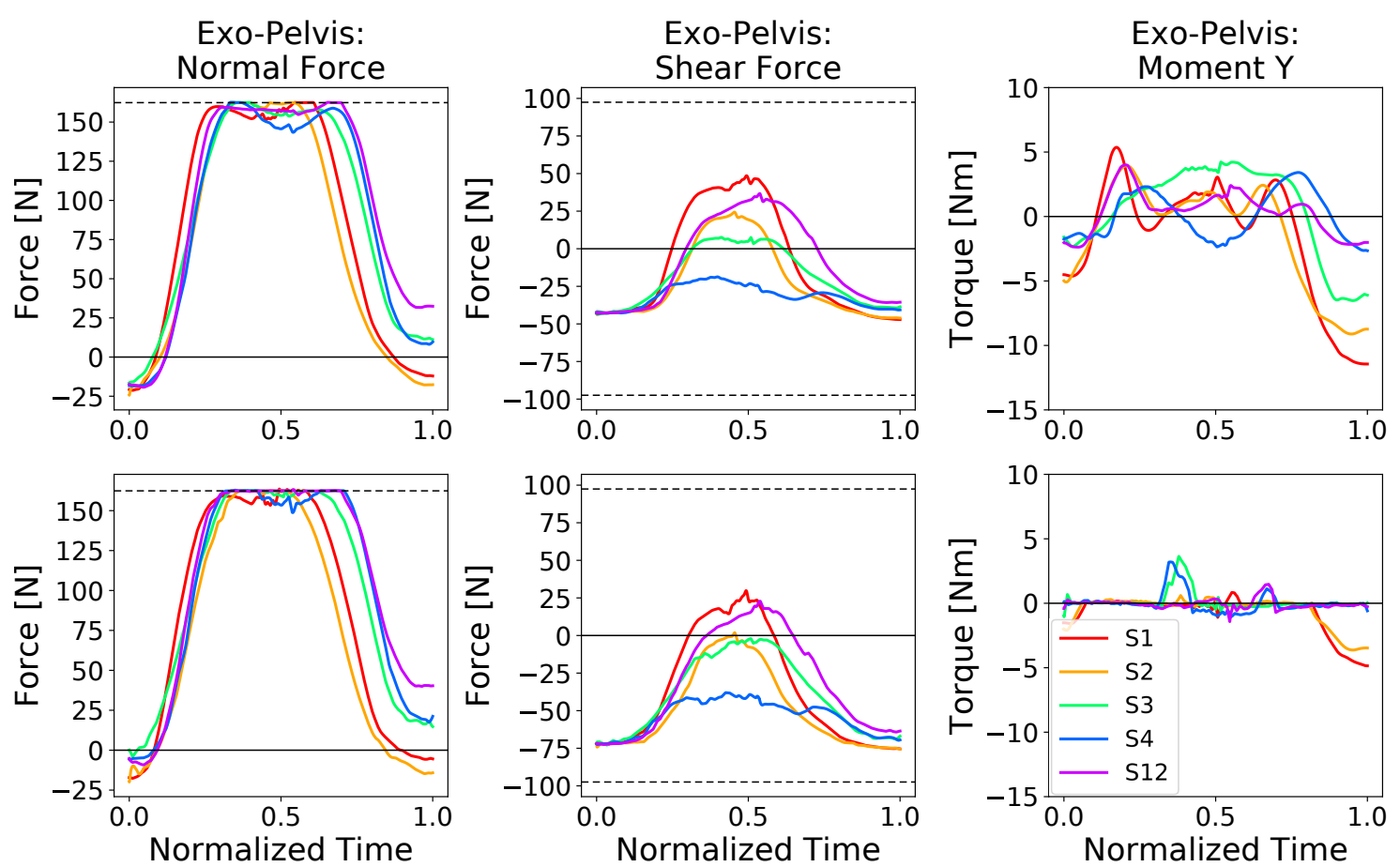

Figure 4. The calculated contact forces and moment acting at the pelvis contact point using the exoskeleton configuration of PO (first row) and of DC (second row). The dashed lines indicate the prescribed limits on the forces during the optimization.

\section{REFERENCES}

[1] Park, J.H., Stegall, P.R., Roye, D.P., Agrawal, S.K.: Robotic spine exoskeleton (rose): Characterizing the 3-d stiffness of the human torso in the treatment of spine deformity. IEEE Transactions on Neural Systems and Rehabilitation Engineering 26(5) (2018) 1026-1035

[2] Jarrassé, N., Proietti, T., Crocher, V., Robertson, J., Sahbani, A., Morel, Guillaume andRobyBrami, A.: Robotic exoskeletons: a perspective for the rehabilitation of arm coordination in stroke patients. Frontiers in human neuroscience 8(947) (2014)

[3] Aach, M., Cruciger, O., Sczesny-Kaiser, M., Höffken, O., Meindl, R.C., Tegenthoff, M., Schwenkreis, P., Sankai, Y., Schildhauer, T.A.: Voluntary driven exoskeleton as a new tool for rehabilitation in chronic spinal cord injury: a pilot study. The Spine Journal 14(12) (2014) $2847-2853$

[4] Huysamen, K., de Looze, M., Bosch, T., Ortiz, J., Toxiri, S., O’Sullivan, L.W.: Assessment of an active industrial exoskeleton to aid dynamic lifting and lowering manual handling tasks. Applied Ergonomics 68 (2018) 125-131

[5] Koopman, A.S., Näf, M., Baltrusch, S.J., Kingma, I., Rodriguez-Guerrero, C., Babič, J., de Looze, M.P., van Dieën, J.H.: Biomechanical evaluation of a new passive back support exoskeleton. Journal of Biomechanics 105 (2020) 109795

[6] Luo, Z., Yu, Y.H.: Wearable stooping-assist device in reducing risk of low back disorders during stooped work. 2013 IEEE International Conference on Mechatronics and Automation (2013) 230-236 
[7] de Looze, M.P., Bosch, T., Krause, F., Stadler, K.S., O?Sullivan, L.W.: Exoskeletons for industrial application and their potential effects on physical work load. Ergonomics 59(5) (2016) 671-681 PMID: 26444053.

[8] Zhang, J., Fiers, P., Witte, K., Jackson, R., Poggensee, K., Atkeson, C., Collins, S.: Humanin-the-loop optimization of exoskeleton assistance during walking. Science 356(6344) (2017) $1280-1284$

[9] Näf, M.B., Koopman, A.S., Baltrusch, S., Rodriguez-Guerrero, C., Vanderborght, B., Lefeber, D.: Passive back support exoskeleton improves range of motion using flexible beams. Frontiers in Robotics and AI 5 (2018) 72

[10] Millard, M., Sreenivasa, M., Mombaur, K.: Predicting the motions and forces of wearable robotic systems using optimal control. Frontiers in Robotics \& AI 4 (2017)

[11] Harant, M., Millard, M., Šarabon, N., Mombaur, K.: Cost function evaluation for optimizing design and actuation of an active exoskeleton to ergonomically assist lifting motions. In: IEEE/RAS International Conference on Humanoid Robots (Humanoids 2019). (2019)

[12] Harant, M., Näf, M.B., Mombaur, K.: Optimal control to facilitate the development process of exoskeletons. In: Progress in Industrial Mathematics at ECMI 2021. (2021) submitted

[13] Näf, M.B., Koopman, A.S., Rodriguez-Guerrero, C., Vanderborght, B., Lefeber, D.: Impact of the misalignment compensation mechanism on the relative motion of the spexor backsupport exoskeleton. submitted to IEEE Transactions on Medical Robotics and Bionics (2021)

[14] De Leva, P.: Adjustments to Zatsiorsky-Seluyanov's segment inertia parameters. Journal of Biomechanics 29(9) (1996) 1223-1230

[15] Millard, M., Kleesattel, A.L., Harant, M., Mombaur, K.: A reduced muscle model and planar musculoskeletal model fit for synthesis of whole body movements. Journal of Biomechanics 89 (2019) 11-20

[16] Vanderborght, B., Tsagarakis, N.G., Semini, C., Van Ham, R., Caldwell, D.G.: Maccepa 2.0: Adjustable compliant actuator with stiffening characteristic for energy efficient hopping. In: 2009 IEEE International Conference on Robotics and Automation. (2009) 544-549

[17] Beléndez, T., Neipp, C., Beléndez, A.: Large and small deflections of a cantilever beam. European Journal of Physics 23(3) (may 2002) 371-379

[18] Cho, K., Kim, Y., Yi, D., Jung, M., Lee, K.: Analysis and evaluation of a combined human - exoskeleton model under two different constraints condition. In: Int. Summit on Human Simulation. (05 2012)

[19] Kozinc, Ž., Babič, J., Šarabon, N.: Human pressure tolerance and effects of different padding materials with implications for development of exoskeletons and similar devices. Applied Ergonomics 93 (2021) 103379

[20] Winters, J.M., Stark, L.: Estimated mechanical properties of synergistic muscles involved in movements of a variety of human joints. Journal of Biomechanics 21(12) (1988) 1027-1041

[21] Leineweber, D., Schäfer, A., Bock, H., Schlöder, J.: An efficient multiple shooting based reduced SQP strategy for large-scale dynamic process optimization: Part II: Software aspects and applications. Computers \& Chemical Engineering 27(2) (2003) 167-174

[22] Felis, M.L.: Rbdl: an efficient rigid-body dynamics library using recursive algorithms. Autonomous Robots 41 (2017) 495-511 